\title{
Variations
}

Variations

Revue internationale de théorie critique

$23 \mid 2020$

Pour une gauche érotique

\section{Quand la Covid dévoile le principe de rationnement}

\section{Arnaud Le Marchand}

\section{OpenEdition}

Journals

Édition électronique

URL : http://journals.openedition.org/variations/1767

DOI : 10.4000/variations. 1767

ISSN : 1968-3960

Éditeur

Les amis de Variations

Référence électronique

Arnaud Le Marchand, "Quand la Covid dévoile le principe de rationnement », Variations [En ligne], 23 | 2020, mis en ligne le 01 septembre 2020, consulté le 07 septembre 2020. URL : http:// journals.openedition.org/variations/1767 ; DOI : https://doi.org/10.4000/variations.1767

Ce document a été généré automatiquement le 7 septembre 2020

Les ami•e•s de Variations 


\title{
Quand la Covid dévoile le principe de rationnement
}

\author{
Arnaud Le Marchand
}

1 Lors d'un discours prononcé à Bastia, le 7 février 2018, alors qu'il récuse la mesure visant à créer un statut de résident restreignant l'accès au marché de l'immobilier sur la Corse, le Président Emmanuel Macron prononce les mots suivants: «Elle ne permettrait pas de régler le problème de fond, elle conduirait à un rationnement, la flambée des loyers et je ne connais aucune économie dans le monde qui ait prospéré sur cette base, aucune. » Dans cette phrase, se lit le rejet libéral du rationnement, qui ne peut être qu'une disposition transitoire toujours moins efficace qu'une pure allocation marchande, à savoir une régulation par les prix de l'offre et de la demande. La crise du Covid-19 va ramener ce concept dans le débat public. Le 19 mars 2020, «Coronavirus : la pénurie de masques, grande colère des soignants" titre Le Monde: «Le rationnement au compte-gouttes de cet équipement de protection suscite l'incompréhension dans les hôpitaux et les cabinets médicaux libéraux ", peut-on lire dans l'article. On n'avait parlé jusque-là que de confinement. Mais de fait, il amenait directement au rationnement, y compris alimentaire, et de façon pandémique, puisqu'une part croissante de la population mondiale risque d'en être affectée.

Si le terme est associé à l'économie de guerre, ou aux critiques du socialisme, ce fut l'un des éléments centraux de la théorie du déséquilibre, à court terme (Malinvaud, 1980, p 49). Dans cette construction, le rationnement provenait de la rigidité des prix. Depuis, les débats autour de l'écologie ont rappelé la méthode du rationnement publique, et la possibilité du choix de l'auto-rationnement (Szuba et Semal, 2010). Car le rationnement peut résulter de politiques, ou de l'application de règles, dont le plafonnement des prix ne sera qu'une conséquence, éventuellement secondaire. Cette question du rationnement est présente dans les débats sur les économies morales, qui organisent le partage des subsistances selon des valeurs dont la transgression amène à des résistances et des révoltes (Thompson, 1971). Edward Thompson y oppose clairement rationnement et restriction par la hausse des prix. Des consommateurs ou des offreurs peuvent ainsi refuser un prix de marché, compatibles avec leurs budgets, qu'ils 
jugeraient cependant anormal. Le thème se retrouve dans l'étude des codes de famine, dont les défauts, ou l'inapplication, aggravent les conséquences des pénuries (Sen, 1977 ; Ellman, 2000). Deux économistes critiques, Pétronille Rème Harnay, et Bernard Guerrien, dans un article publié par les Cahiers internationaux de sociologie, citaient les règles de rationnement, parmi les implicites et les impensés de la théorie économique. Les conséquences du Covid-19, telles des pénuries réelles et spectaculaires, incitent à repenser le rationnement, c'est à dire le schéma, les principes, selon lesquels on répartit cette pénurie. Mais ces principes existent aussi dans les périodes normales, pour prendre des décisions quand l'offre et la demande divergent, ou sont perçues comme divergentes. La théorie post-keynesienne étudie explicitement la possibilité d'un rationnement du crédit (Asensio et al., 2011). Cette possibilité ne se limite pas au crédit, elle existe aussi sur les marchés de biens. Quant au chômage keynesien, il est une forme de rationnement de salariés.

3 Les hypothèses sont les suivantes: Il y a des principes de rationnement, plus ou moins cachés, dans le fonctionnement des échanges, pour prendre des mesures face à une impossibilité de satisfaire la demande ou l'offre par un seul mouvement de prix. Dans une économie de marché, leur mise en œuvre est, dans les périodes de paix, décentralisée. Ces règles sont souvent propres à un lieu ou à un milieu, dont elles reflètent en partie les divisions sociales. Car ces règles portent d'abord sur les personnes: elles disent qui est rationné et dans quelles proportions, d'où leurs dimensions sociales. Elles sont plus faciles à mettre en lumière autour des marchés physiques, dont font partie les marchés à terme. Un examen de l'exemple historique fourni par le rationnement, en tant que pratique et en tant que concept, lors du siège de Paris de1870-71, montre son rejet dans le diagramme libéral.

Quoique locales, les règles de rationnement sont hiérarchisées, celles du crédit par exemple, s'imposant à d'autres marchés. Alors que le rôle de la monnaie était neutralisé dans la théorie du déséquilibre, au contraire, l'hétérogénéité et la pluralité monétaire (Zelizer, 2005, Théret 2008) participent de ces règles. Ainsi, si les tickets de rationnement sont des compléments monétaires (Blanc, 2000), les monnaies complémentaires peuvent être adoptées pour organiser un rationnement sans accord de l'État. Le contingentement de certains acteurs permet, de façon plus paradoxale, de légitimer des constructions, comme les marchés à terme à partir de la fin du XIXe siècle, via cette pluralité. Car si l'on peut penser des monnaies alternatives, visant à sortir du capitalisme (Fourel 2019), les monnaies complémentaires historiques sont plutôt des outils dans les interstices de l'économie marchande. Ces règles ont des dimensions spatiales, que la crise du Covid-19 a rendu visibles, sur les marchés physiques. En outre, il y a transmission des marchés de biens aux salariés en quête d'emploi. Le travail précaire ou saisonnier est ainsi une forme de travail rationné.

\section{Invention et déni du rationnement dans le diagramme libéral : le siège de Paris}

5 Le terme rationnement serait entré dans le français courant à l'occasion du siège de Paris, lors de la guerre franco-prussienne de 1870. Cette causalité initiale explique sans doute qu'on l'associe à l'économie de guerre. Pourtant, à cette occasion, la seule mesure prise immédiatement fut le moratoire des loyers et des dettes. 
6 Jacques Henri Paradis, dans son Journal du siège de Paris, décrit, par des observations quotidiennes, la juxtaposition de principes différents, à la fois la restriction marchande, par les hauts prix, et un rationnement pour les plus démunis. Ce double mécanisme des échanges produit une désorganisation, qui aboutit à des émeutes à la fin du siège. Il observe qu'à partir de la fin janvier, la hausse des prix n'est plus légitime. Ainsi pour le 28 janvier (Paradis, 2010, p. 374):

«La prétention exorbitante d'une vendeuse de pommes de terre a été le signal des incidents regrettables qui se sont produits. Une marchande qui réclamait 45 francs pour trois lapins les a vus enlevés contre un payement de 15 francs. Pendant quelque temps le mouvement populaire s'est borné à rectifier de la sorte les prix demandés. Ensuite le désordre augmentant, on a vu des individus de mauvaise mine se substituer d'autorité aux marchands, et vendre à bas prix, puis donner, puis enfin jeter à tour de bras toutes espèces de denrées... »

7 Un témoignage similaire est donné par Adolphe Michel, dans son journal (Michel 2012, p. 221), qui constate que les ventes directes se substituent aux marchés : ces derniers ne produisent plus de cours, il n'y a plus de prix moyen.

8 Hyppolite Lissagaray, témoin à l'autre extrémité du champ politique, relie le déclenchement de la Commune à la fin du moratoire des loyers et des échéances, le 10 mars 1871, alors que les artisans et les locataires ont été privés de ressource du fait du siège. La non-compensation de leur rationnement par le report des dettes est une des causes du soulèvement (Lissagaray, 1970, p. 104). Ce conflit autour du logement ne sera pas sans suite. Danièle Voldman (Voldman, 2016) note que la préfecture de Paris, dès 1873, obtient des fonds pour aider les locataires en difficulté. En fait, il y a bien une légère irréversibilité : on ne peut revenir au statu quo d'avant le siège.

Or la position des autorités fut réaffirmée dès 1872, en réponse aux débats que soulevaient encore ces questions. Le président de la commission parlementaire, le comte Daru, conclura son rapport par cette vision définitive :

«Nous tenons pour certain que le rationnement, surtout dans une population civile nombreuse amènera toujours le gaspillage, à moins que la ration ne soit extrêmement réduite. Au contraire, tant que le rationnement n'existe pas, chacun n'achète que ce qu'il consomme et il y a peu de pertes. » (Daru, 1873).

10 Le rejet du rationnement dans le diagramme libéral est bien antérieur au socialisme du XXe siècle. Il est en fait un déni.

\section{Le rationnement du crédit}

11 Aujourd'hui, en dehors de l'économie de la santé, le rationnement du crédit semble seul retenir l'attention des économistes, notamment pour les PME en France (Alexandre et Buisson-Stephan, 2008). La position classique est de l'analyser comme résultant d'un problème d'information, qui élimine les entreprises les plus difficiles à évaluer ou ayant le moins de trésorerie. Cependant, le rationnement préventif du crédit peut être pratiqué par les banques, qui, anticipant une récession sur un marché, éliminent certain des concurrents avant que la crise ne se manifeste. Le rationnement du crédit est donc une fonction essentielle des banques à la régulation de la concurrence et à la structuration d'un secteur. Cela implique, a contrario, une responsabilité des banques dans la création de surcapacités. Le rationnement est bien une condition du fonctionnement normal d'un marché, rendu d'autant plus nécessaire que le cycle des affaires y est plus marqué, ou que l'entrée y est plus facile. C'est aussi pour faire face à 
ces rationnements du crédit qu'ont pu être justifiés, entre autres raisons, la mise en place de monnaies complémentaires. L'adoption de ces mêmes monnaies peut aussi reposer sur l'acceptation d'un rationnement volontaire des échanges possibles. Ainsi dès le xixe siècle, le rationnement du crédit avait été l'occasion de réorganiser les marchés à terme, via la pluralité monétaire.

\section{Caisse de liquidation et légitimation des Marchés à terme}

Marieke de Goede (de Goede, 2005) a montré l'importance dans la généalogie de la finance, de la levée de l'exception de jeu contre les marchés à terme. C'est à dire du soupçon d'assimilation de ces marchés à une forme de loterie. Or, pour obtenir la suppression de cet obstacle, les négociants vont organiser une forme de clôture et de rationnement du marché, sous la forme d'une caisse de liquidation, le clearing fund, qui fait intervenir une monnaie de compte. Celle-ci est une monnaie endogène, propre à la place. Cette innovation interviendra sur les marchés à terme du Havre, pour le café et le coton, à l'occasion d'un credit crunch. En effet, le krach de l'Union Générale, en janvier 1882, a précipité des faillites bancaires et une contraction des dépenses publiques, les crédits bancaires sont plus restreints, et ce jusqu'à la fin du siècle (Bouvier, 1960). Le marché à terme du café, au Havre, va pourtant se transformer et deviendra l'un des plus importants du monde (Malon, 2017) jusqu'à la Première Guerre mondiale. Ce marché se dote d'une caisse de liquidation en novembre 1882, puis adopte les règlements définitifs des opérations à terme, en 1883. La caisse de liquidation est l'adoption d'une monnaie de paiement scripturale, à usage local : les transactions s'effectuant via des jeux d'écritures avant le solde, seuls les écarts par rapport aux cotes prévues donnent lieu à des paiements en monnaie métallique. Si l'on avait parfois recours à cette technique de façon transitoire sur le marché des actions (Bouvier, 1960), elle n'était pas contrôlée par une instance de régulation. Celle-ci achève la création d'une monnaie à usage limité, à ce marché. Elle ne compte que jusqu'à la livraison, quand la marchandise quitte l'entrepôt et le marché, pour un utilisateur final. Ce dispositif permet de s'adapter à un manque de capitaux. De surcroît, dans l'étude des marchés à terme qu'il publie en 1888, Olivier Senn, négociant havrais, argue que, lors du krach, l'invocation de l'exception de jeu a été redoutée. L'exception de jeu pouvait être invoquée pour obtenir l'annulation d'un contrat, qui semblait rétrospectivement reposer sur un pari incertain, un agiotage. On pouvait annuler un contrat, si le vendeur ne possédait pas encore la marchandise, ou le titre, au moment de sa passation. Senn note que :

« Après le krach de 1882, la question... n'avait jamais cessé d'être à l'ordre du jour, il semble que plusieurs agents de change qui avaient craint de se voir opposer par leurs clients, spéculateurs à la hausse, l'exception de jeu, aient refusé les ordres d'achat et que la baisse s'en soit trouvé précipitée, aggravée » (Senn, 1888, p. 174).

Pour Senn, la solution est la caisse de liquidation, car en garantissant le sérieux des opérations, elle permet d'assurer que les opérations à termes ne sont pas des paris. La caisse de liquidation, par les garanties qu'elle apporte, et surtout parce qu'elle permet de ne pas engager de liquidités, transforme ces opérations en les éloignant du jeu. C'est de cette façon que les négociants obtiendront la légitimation en 1885. La caisse est organisation de la création d'une monnaie endogène réservée, qui sert à refonder et à justifier le marché, à tel point que, pour certains négociants, la caisse et le marché à terme seront des synonymes. La méthode sera copiée à Paris, en 1911 (Lacombe, 1938) 
bien après New York, où l'on on adoptera la method of clearing « du Havre » dès 1885 (Norman, 2011). Si l'on suit la présentation du fonctionnement de cette caisse donnée par Robert Lacombe dans sa thèse, en 1939, le marché devient un club de spécialistes. Ce dispositif filtre les opérateurs n'ayant pas suffisamment de technique ou de crédit. Ce point est clairement présenté par Senn: il n'hésite pas à employer le terme de corporations pour désigner le club des négociants sur ce marché. Il y a donc continuation d'un encastrement dans un réseau social du négoce, à la fois local et international (Bartolomei et alii 2017), qui justifie le rationnement des non-membres.

En effet, une fois établie la liste des membres, la caisse de liquidation offre à la corporation « la possibilité de faire plus d'affaire avec les mêmes capitaux » (Senn 1888, p. 110), grâce à la monnaie endogène. La littérature économique, dès 1912, admet qu'elle accroit la vitesse de circulation des capitaux sur le marché et compense ainsi leur relative rareté (Turmann, 1912). Mais la caisse de liquidation peut, en modulant le montant du dépôt préalable de garantie (le déposit), réduire la monnaie sur le marché pour freiner la spéculation. Le marché à terme et la caisse ont aussi pour but d'empêcher les étranglements, c'est-à-dire les coalitions d'acteurs visant à accentuer une hausse des prix. Le déposit peut alors être augmenté pour contrecarrer les prises de position jugée hostiles. La caisse peut ainsi rationner les négociants étrangers. Les Brésiliens s'en plaignent dans le journal du Havre (Journal du Havre, 9 et 10 octobre 1887). De fait, ce sont les marchés de New-York et du Havre qui fixent les prix du café (Topik, 1991, p. 437). En outre, le Bulletin de la bourse du Havre annonce le 18 novembre 1890, la possibilité pour un opérateur du marché à terme de livrer en place du café qualité Santos, des cafés d'autres provenances et même de surseoir d'un mois ou plus à une livraison prévue par contrat. L'avantage est de pouvoir résister à une réduction de l'offre de café Santos, malgré des contrats déjà engagés. Le Bulletin remarque dans son édition du 24 novembre 1890 que cela revient à reconnaitre que les opérations à terme ne sont pas toujours réelles, et donc à retomber sous le coup de l'exception de jeu, puisqu'il s'agirait de vendre une marchandise sans être certain de la fournir. Ce changement de règle n'avait pas un soutien unanime, parmi les négociants. Certains, dont le bulletin se fait l'écho, craignent que l'Etat ne reviennent sur l'exception de jeu et interdisent les marchés à terme. Elle sera pourtant définitivement adoptée en 1896, alors que Senn avait, en 1888, pris soin de rappeler que sur un marché à terme, le vendeur est dans l'obligation de livrer, pour bien éloigner l'assimilation aux paris. La légitimation des opérations à terme a donc été obtenue par la fermeture du marché, c'est à dire par un schéma de rationnement, et non pas par la démonstration de l'efficience informationnelle (Walter 2005).

$\mathrm{Ni}$ cette institution, ni la justification générale de la finance à laquelle elle va contribuer, ne sont le fruit d'un mécanisme sans sujet. Une approche institutionnaliste $\mathrm{du}$ rationnement, et de la monnaie endogène, sont nécessaires pour les comprendre (Laurentjoye et Malherbe 2020). Au-delà de la sphère financière, ce rationnement s'appliquait aussi aux travailleurs de la filière portuaire.

\section{Jetons pour travailleurs pauvres}

Dans le marché à terme, est donc utilisée une monnaie de compte, comme moyen de limiter les risques et la concurrence, cette solution était aussi présente sur le marché du travail de la manutention. En effet, une monnaie de convention, pour compenser un 
manque de capitaux des entrepreneurs qui ne peuvent faire l'avance des salaires, est utilisée pour le paiement des dockers journaliers, sous forme de jetons, échangeables dans des cafés du port contre des francs, puis par les débitants de boisson auprès des employeurs. Ces derniers les rachètent aux débitants, une fois que les armements les ont payés. Cet usage remontait au moins à 1870. Ces pièces de cuivre portaient les marques des compagnies d'armement ou de manutention, comme celles que l'on achetait dans les maisons closes pour payer les tenancières, au moins depuis le Second Empire (Lerquet, 2014). Cette pratique existait aussi aux USA (Zelizer, 2005, p. 169-170). Dans ce cadre, où les prostituées ne touchaient pas de salaire, les jetons servaient de certificat de travail intérieur au dispositif. Les prostituées, auxquelles était imputée à leur entrée une dette fictive, les remettaient pour preuve, et ne percevaient que des pourboires. Ce qui leur rend presque impossible la sortie de la maison close. Ces jetons ont donc une face dans laquelle ils compensent un manque de crédit, et une autre, par laquelle ils sont un moyen de rationner, ou de maintenir dans le travail forcé, les travailleurs les plus vulnérables. Dans les campagnes, ce sont des mauvaises monnaies de cuivre (Thuiller, 1959) que l'on utilisait, à cause de la rareté du numéraire en argent, pour servir aux salaires des brassiers, mais ces billons avaient un cours légal.

17 Cette monnaie complémentaire renforce, par le choix des cafés comme lieu d'échange, la marque d'infériorité sociale. De fait, c'est au bagne de Nouvelle-Calédonie par exemple, que l'on paie des salaires en alcool, ou en tabac, aux déportés, que l'on pratique des retards dans les paiements en monnaie, ainsi que le rapporte dans ses mémoires François Cron (Cron, 1989, p. 157-161, 166, 199-202). Ce type de monnaie complémentaire est utilisé aussi là où on ne l'attend pas, ainsi en 1863, dans le familistère du Guise. Godin tente de s'en servir pour discipliner les ouvriers "immoraux", c'est à dire pour contrôler les dépenses, car les jetons ne sont échangeables qu'à l'épicerie du Palais social. Mais les jetons du familistère furent l'objet de contrefaçons (Lallement, 2009, p. 146). Or, le 7 octobre 1892, le Journal du Havre, sous l'intitulé «faux-jetons » relatait le fait divers suivant : Un journalier avait présenté 4 jetons en cuivre estampillés Compagnie générale Transatlantique à un débitant de boisson, qui avait accepté de les changer contre des francs, avant de s'apercevoir qu'ils étaient faux. Ils avaient donc bien un usage assez courant, pour que l'on refuse la contrefaçon, de ce qui était déjà de la fausse monnaie.

Ces jetons fut plusieurs fois l'enjeu de grèves dès 1887. En 1888, une demande d'intervention auprès du Sous-Préfet avait été rejetée au motif que les jetons constitueraient des certificats de travail, non de la monnaie complémentaire. Ce qui l'assimile implicitement aux jetons-monnaies des maisons closes. Une nouvelle grève dont les jetons sont l'un des enjeux a lieu en 1892-93. Cette grève intervient à l'automne, alors que la ville du Havre a connu une épidémie de choléra, durant l'été (Vigarié 2003). Celle-ci a fait plus de 500 morts, le trafic portuaire a été en partie arrêté. Ce choc sanitaire a des conséquences économiques : une diminution des liquidités, un ralentissement de l'activité, et un recours aux jetons comme moyen de paiement des dockers. La revendication initiale de la grève est une augmentation du salaire journalier, de 5 à $6 \mathrm{~F}$, pour compenser l'intermittence, habituelle et exceptionnelle, en quelque sorte endémique, mais les revendications comprennent aussi la disparition des jetons. La mairie se tourne alors vers la Chambre de Commerce. Elle demande si la Chambre pourrait « établir des bureaux spéciaux où les jetons seraient échangés contre du numéraire et qui se substitueraient fort avantageusement, aux endroits dans 
lesquels se font actuellement les opérations de cette nature » (courrier le 24/10/1892). Ce qui revient implicitement à demander à la Chambre de commerce d'organiser une bourse du travail, sur laquelle la Mairie aurait alors un droit de police. La chambre décline par crainte de ne pas contrôler la solvabilité des bons et par peur de voir la pratique s'étendre. Elle est donc bien consciente du problème. Aussi le 23 novembre 1892, la Mairie doit faire appel « au concours moral des entreprises de déchargement, mais aussi aux négociants pour lesquels ils travaillent, de renoncer à ce mode de paiement et de crédit ». Dans une délibération du conseil municipal du Havre le 9 décembre 1892, dans lequel la question sociale a fait son entrée, il est explicitement rappelé que les jetons des entrepreneurs n'ont aucune valeur légale, qu'un ouvrier les ayant perdus a toujours droit à son salaire. Mais il est aussi concédé qu'ils constituent un capital de roulement pour des entreprises ayant des capitaux restreints. Lors d'une réunion tenue le 26 décembre, en présence du Préfet, du Maire du Havre et du sousPréfet $^{1}$, promesse est faite par les négociants et entrepreneurs de faire cesser le paiement en jetons, qui renforçait symboliquement l'infériorité sociale des ouvriers. Le climat politique n'est sans doute pas étranger à ce revirement : en décembre 1887 la grève des ouvriers du port n'avait pas eu les honneurs de la presse locale, mais à partir de janvier 1888, après que Louise Michel ait été victime d'une tentative d'assassinat lors d'un meeting au Havre, l'agitation anarchiste et les risques d'attentats allant croissant, la presse locale se préoccupe davantage des problèmes sociaux. Au niveau national, le retour des grèves est aussi une conséquence du Krach de 1882 (Perrot 1973). Les jetons seront donc interdits par l'Etat en 1894. Ils sont néanmoins réapparus plusieurs fois, en 1895, 1916 et 1925. La municipalité se déclarait impuissante face à une pratique monétaire, qui n'était pas de ses compétences et que l'Etat, en détournant ses yeux, tolérait car elle accompagnait la croissance du commerce maritime. En 1893, la préfecture avait seulement suggéré de les remplacer par des billons. Ces épisodes à durée limitée, mais récurrents, d'utilisation de monnaies complémentaires étaient en partie assimilables à une épidémie, même sans l'intervention d'un virus ou d'un bacille : ils concernaient une population vulnérable, qui était contrainte de les accepter, parce que les employeurs manquaient de liquidités, en attendant une meilleure conjoncture.

Le rationnement du crédit se prolonge pour certains acteurs, les moins bien dotés, les plus bas dans la hiérarchie sociale, en paiement dans une monnaie inférieure. Ce phénomène peut prendre une forme spatiale, qui est plus visible sur les marchés physiques.

\section{Le rationnement sur les marchés physiques}

\section{Files d'attente et marchés de plein air}

La file d'attente est l'un des exemples souvent cités d'ordre émergent, pris dans une boucle entre pratiques individuelles, conventions d'attentes réciproques et appel implicite ou explicite à des règles morales plus globales (Kaufmann et Quéré, 2001). Mais elle émerge dans un cadre contraint et spatialisé. Le 16 mars 2020, les gens, dans la queue devant un supermarché, commentent le confinement et ce qu'ils ont vus dans d'autres magasins. Certains ont patienté trois heures, avant d'entrer, pour cause de limitation à $100 \mathrm{du}$ nombre des clients admissibles à la fois. Or, les entrées sont limitées, mais pas les achats : les premiers arrivés font des stocks, jusqu'à 25 plaquettes de margarine, des dizaines de bouteilles d'huile, etc. Ce public tire la première 
conclusion : si les entrées sont contingentées, il faut plafonner les achats. En Belgique, les grands magasins limitent les achats à un produit par type et par famille. Le 18 mars, le journal Le Monde indique que certaines grandes surfaces réservent certains créneaux horaires aux "seniors». Ce qui est rationné, ce ne sont pas des produits, mais des personnes.

En temps normal, il y a une organisation spatiale de l'accès et de la concurrence sur les places de marché et les foires. Ces regroupements de petits commerçants itinérants illustrent une forme de concurrence presque parfaite. Avant cet apparent équilibre, une répartition qui peut être conflictuelle, est opérée par la municipalité pour régler l'attribution des places. Les places ne sont pas équivalentes dans l'accès qu'elles procurent à la clientèle. Il $\mathrm{y}$ a de bons et de moins bons emplacements. Les règles d'attribution de ces places font l'objet de négociations. On peut envisager les enchères, le droit d'ancienneté, ou le tirage au sort. Si les enchères constituent une solution par les prix, elles sont considérées comme la solution la moins facile à mettre en œuvre. Les enchères peuvent monter trop haut, générer des surcoûts, exclure les nouveautés. La préférence des marchands va donc vers un contingentement, éventuellement mixant plusieurs pratiques (ancienneté et hasard par exemple). On peut aussi partager l'espace entre plusieurs règles. Le problème est de trouver une répartition des acteurs qui satisfasse les clients, et permette un renouvellement pour garder l'attractivité de la manifestation. Pour que le rassemblement et la rencontre entre vendeurs et acheteurs se déroulent bien, un principe de rationnement de l'espace doit être dégagé. C'est un préalable à la fixation des prix, qui peut vite être déstabilisé par une crise sanitaire. Mais ce n'est pas le seul, comme les marchés au cadran permettent de l'observer.

\section{Marchés parfaits face aux épidémies : les saisonniers rationnés}

Sur les marchés au cadran, les transactions ont lieu à l'équilibre, via l'organisation des enchères, ce qui exclut, a priori, le rationnement selon la théorie néo-classique (Tricou, 2008). Pourtant, ces marchés contiennent de façon implicite, un principe de rationnement, jusqu'à ce qu'une crise le dévoile. En cas de désaccord, une crise institutionnelle peut alors advenir et devenir déconstruction sociale du marché. Le marché au porc breton est d'autant plus éclairant sur ce point, que le secteur est périodiquement affecté par des épidémies, qui peuvent être internationales (DEP, Peste porcine), et qui se traduisent par des embargos sanitaires, en sus des embargos politiques. Autour du marché au cadran de Plérin, en 2015, s'est joué une crise de la construction sociale du marché. La présence de la peste porcine africaine déclenche un embargo sanitaire russe, et une mévente, il y a alors conflit entre acheteurs et vendeurs sur la façon de répartir le rationnement. Selon le règlement du marché de Plérin, l'ordre de passage des vendeurs est fixé par le tirage au sort, ce qui est un premier principe de répartition des chances de vente. Puis en fin de séance, les lots invendus à un prix en deçà de 6 centimes au-dessous du prix moyen, sont attribués aux acheteurs inscrits, au prix moyen de la séance, dans les limites des quotas prédéfinis. C'est une règle qui limite la possibilité pour les acheteurs d'acquérir au-dessous de ce prix moyen. Sa finalité productiviste est dénoncée au moment de la crise. Les acheteurs, et notamment l'entreprise Bigard et la coopérative Cooperl, quittent le marché, qui n'a plus de cotation pendant sept semaines, d'octobre à novembre 2015. C'est sous cette menace que certains producteurs vont accepter de renégocier des baisses de prix ${ }^{2}$, malgré le soutien actif du ministère de l'agriculture au marché et aux prix. Pour autant 
le même problème se repose en 2016 et encore en février 2018, alors que le volume des ventes, sur le marché au cadran, a été divisé par deux par rapport à la décennie précédente. Ce qui peut faire dire au président du comité régional porcin: "Nous sommes une génération qui n'a pas connu l'avant-marché, mais qui ne sait surtout pas ce que pourrait être l'après... » dans Le Paysan Breton du 14 mai 2018. Ce marché au cadran n'était pas un lieu régi par la seule loi de l'offre et de la demande. Mais l'application de cette seule loi semble conduire à son remplacement par la régulation des grandes entreprises et à une montée de l'incertitude pour les petits producteurs. Les acteurs en charge de la direction du dispositif vont alors infléchir leurs discours. Si dans les années 1980 et 1990, les références aux analyses néo-classiques étaient nombreuses et contribuaient à former les pratiques, au début du xxi siècle, on voit la réutilisation de la critique épistémologique des marchés parfaits. Le directeur, au cours d'un entretien téléphonique, en juin 2018, assigne aux règles de fixation des prix, le rôle d'aider à faire vivre ce qu'il appelle : « la convention du marché » L'attribution des lots garantit un approvisionnement aux abattoirs, pour éviter une destruction de la filière, suite à une chute des prix. La règle de rationnement est donc justifiée comme un élément essentiel, qui au cours de l'entretien sera distinguée des prix politiques, c'està-dire fixés de l'extérieur du marché, par l'administration. Pourtant le rôle du rationnement est bien de fixer une fourchette de prix locale, ce qu'il ne parvient que difficilement à faire en cas d'embargo. Un choc épidémique ne crée pas le schéma de rationnement, mais il peut le modifier, en changeant son échelle. Ces chocs épidémiques se répercutent dans la production, et suscitent une demande de travail intérimaire dans les abattoirs de bovins (Muller, 2008), comme de porcs. Le covid-19 lui crée d'abord un nouveau contingentement de l'offre par les fermetures des restaurants. Ensuite, le développement de l'épidémie dans les abattoirs aux USA et en Allemagne fait baisser la production. Celle-ci se propage aussi dans les abattoirs français, provoquant l'arrêt presque total de l'abattoir de Kerméné, dans les dernières semaines de mai 2020. Pour autant les prix ne montent pas, car la demande reste faible: il n'existe donc pas réellement de pénurie de main d'œuvre pour les employeurs, puisque leurs débouchés ont chuté eux aussi ( $\left.{ }^{3}\right)$. C'est toujours le même régime de restrictions, et d'insécurités, pour les salariés, que le Covid-19 ne fait qu'intensifier et généraliser, comme d'ailleurs le remarquera Enrico Somaglia, secrétaire général de la fédération européenne des syndicats de l'alimentation, de l'agriculture et du tourisme ( EFFAT) dans EURACTIV du 19 mai 2020 : l'épidémie a "exacerbé des problèmes qui sont déjà bien connus dans le secteur», en particulier en ce qui concerne la sécurité des travailleurs » ${ }^{4}$ ). Mais c'est dans le maraîchage que les conséquences de l'épidémie sur le travail intermittent sont les plus visibles.

Dans l'analyse de la construction sociale du marché au cadran des fraises que donne Garcia (Garcia, 1986), l'organisation de la fluidité des échanges, élimine le problème du report de l'offre d'un produit périssable, et peut supprimer un rationnement des vendeurs ou des acheteurs. Comme on peut stocker les fraises d'un jour sur l'autre, grâce aux techniques du froid, les acheteurs ne sont pas forcés d'acheter et peuvent acquérir seulement une petite quantité. Les offreurs peuvent suspendre la production, s'ils anticipent une baisse des ventes, grâce à l'emploi des journaliers. Ce sont donc eux qui, in fine, sont rationnés. Les deux activités sont suffisamment liées pour que le bureau de recrutement des saisonniers ait été un temps, durant les années 2000, installé au marché au cadran de Sologne, marché qui emploie lui-même des saisonniers pour son propre fonctionnement. 
24 Le marché du travail des saisonniers est fortement segmenté. Son développement résulte en partie de la réduction des possibilités d'auto rationnement par lesquelles les entreprises agricoles familiales maintenaient leur compétitivité (Mesini, Laurent 2015). Les travailleurs détachés qui y sont présents sont usuellement contingentés, le nombre de contrat étant fixés. S'il n'y a pas de monnaies inférieures, comme au XIXe siècle, un système de dette (Decosse 2018), et d'autres dispositifs les rendent disponibles, en limitant les offres auxquelles ils peuvent répondre (Bernardot 2012; Chauvin 2010). Dans le cas des saisonniers intérieurs, une partie a recours au logement en caravanes ou camion aménagés. L'exclusion bancaire qui découle de leur mode d'habitat, a comme conséquence le recours au micro-crédit de l'Adie, et pour d'autres le prêt sur gages, en général contre des objets en or, comme s'ils étaient maintenus dans un régime monétaire métallique (Le Marchand 2018). Ce qui a pour conséquence de freiner les sorties du salariat, et de les garder disponibles, comme appoints. Ce rationnement ordinaire des travailleurs précaires de l'agriculture, a pour but de maintenir des bas prix. Or la crise du Covid-19 va là aussi révéler des tensions. Le 22 mars, on annonce la fermeture des marchés ouverts, ce qui induit rationnement de l'offre de produits, tandis que, dès le 20, plusieurs articles dans la presse, telle Les Dernières Nouvelles d'Alsace, déploraient un manque de bras, car les travailleurs détachés polonais, roumains bulgares, marocains, etc. ne peuvent venir en France. On serait face à une pénurie de main d'œuvre, qui commence à faire craindre pour les récoltes, et envisager un risque de pénurie alimentaire. Le 24, le journal Le Monde titre "L'agriculture a besoin de 200000 saisonniers ». Le groupe Facebook "saisonniers en habitat mobile ", dont l'essentiel des posts est consacré aux annonces d'emploi, aux recherches d'information sur les emplois et aux problèmes de stationnement des camions aménagés, a plus de 17292 membres en avril 2020, soit $82 \%$ des saisonniers en grands déplacements en 2015, hors Gens du Voyage. A partir de la fin mars, ils commentent la situation sur cet hybride entre forum et plate-forme numérique de placement. Selon certains de ses membres, les employeurs refusent d'embaucher à un salaire différent de celui pratiqué pour les travailleurs détachés. Ils dénoncent de fait un rationnement de leur offre par les employeurs et une pénurie illusoire, soit une convention sur l'état du marché, autoréférencée. Ainsi le 29 mars, une des saisonnières s'étonne :

« Je comprends pas, je trouve pas de boulot dans le coin où je suis. En fait, les producteurs perdent leurs débouchés au fur et à mesure, j'ai l'impression. Plus de marché déjà et apparemment des magasins comme Grand Frais perdent leurs ventes et ne renouvellent pas leurs achats. Par exemple, je devais récolter les asperges, car ils avaient pas les Polonais comme d'habitude et en fait, ils me disent que finalement c'est pas la peine que je vienne, car ils ne savent pas ce qu'ils vont faire de $30 \%$ de leur récolte. »

La situation qu'elle décrit correspondrait à un triple rationnement celui de l'offre des biens, celui des employeurs, et celui des travailleurs. Malinvaud considérait comme impossible, ou très peu probable, le double rationnement des entreprises, sur le marché des biens et du travail (Malinvaud 1980, p.71 et p. 134). Dans ce témoignage, ce n'est pas la demande de travail qui est rationnée, mais l'offre des travailleurs, et celle des produits. Dans ce cas précis, le choc épidémique ne dérègle pas le fonctionnement à court terme, il le tend et le révèle.

En fait, Le Monde continue une série d'articles sur les saisonniers, dont le titre est souvent démenti par le contenu : ainsi, le 8 et 9 mai ( $\mathrm{p} 16)$ «Les agriculteurs recherchent toujours des bras». On y lit que "Les exploitants espèrent une ouverture des frontières dans 
l'Espace Schengen pour leurs recrutements. » En outre, dans cet article on apprend que la plate-forme " des bras pour ton assiette, sur WiziFarm » : comptabilise 330000 comptes ouverts et 284000 travailleurs inscrits, or en face, seule 6000 offres en ligne, soit cinquante fois moins que de demandes pour 1400 missions concrétisés commente $\mathrm{Mr}$ Vervy (le responsable de la plate-forme). L'explication de ce décalage passera par le reproche, aux saisonniers locaux, d'un salaire trop élevé et d'une productivité insuffisante. Les entreprises y pallieraient par un partage de main d'œuvre détachée entre elles. Dans ce cas, les employeurs ne sont pas rationnés car ils ne manquent pas de personnel bon marché, ou alors face aux incertitudes sur la demande, ils n'embauchent pas, et ce sont bien les saisonniers qui sont rationnés. Dans Le Monde du 16 mai, p. 8, «Les agriculteurs attendent leurs saisonniers. Malgré les promesses du gouvernement la main d'œuvre reste encore souvent bloquée aux frontières ${ }^{5}$. " L'article est dans la même veine et donne les mêmes chiffres. Par contre le début donne un nouveau sens à la frontière : "La frontière c'est une tombola », dit Javier, un saisonnier espagnol quinquagénaire et expérimenté, c'est l'exact définition d'un rationnement aléatoire. Tandis que la FNSEA justifie ainsi le paradoxe :si on embauche des locaux on ne va pas sortir les récoltes. Elle esquisse alors un risque de destructions de récoltes ou de chances négatives, due à un retard de maturation, qui permet d'attendre avant d'embaucher. En définitive, ce sont bien les salariés qui sont bloqués par le confinement, et non les employeurs, conformément au régime habituel de rationnement. La pénurie de main d'œuvre est une convention, un mantra répété pour ne pas analyser la réalité du fonctionnement en période de baisse d'activité.

\section{Conclusion : technique et divisions sociales}

En 1948, la revue Etudes et Conjoncture, publie un article anonyme sur les techniques de rationnement. L'article n'envisage le problème qu'en cas de pénurie globale, liée à des circonstances exceptionnelles, comme la guerre ou les mauvaises récoltes (les épidémies n'étaient plus envisagées). L'article évoque l'idée théorique d'un rationnement en situation d'abondance, pour améliorer la santé publique. Les techniques de rationnement y sont présentées selon le degré d'abondance du pays et son état d'avancement économique, qui détermine la qualité de l'appareil statistique et celle de l'administration. Certes, pour des raisons techniques, ou en raison de fluctuations spécifiques, certains principes de rationnement sont plus adaptables à certains secteurs qu'à d'autres. Or dans un même secteur, ou un même marché, comme celui de l'intermittence portuaire ou celui des produits de la pêche, plusieurs schémas de rationnement sont possibles, et peuvent parfois même coexister. Car les schémas de rationnement ne différent pas selon les marchés, mais selon les sociétés, et leurs valeurs et leurs divisions. Pour un auteur comme Paul Jorion (Jorion, 2010), les principes de rationnement (il emploie le terme contingentement dans ses études sur la pêche) sont en nombres limités et relèvent d'une anthropologie transhistorique des marchés. Il les analyse en termes de rapport entre des statuts personnels, dans une perspective aristotélicienne. Cependant, on peut aussi montrer des variations historiques du mode de rationnement, à la faveur de contestations par ceux qui le subissent, ou de changements dans les priorités des organisations impliquées.

Dans les économies de marché, en dehors des périodes de crise aiguë, il peut être présenté comme une règle particulière, relative à un lieu ou à un milieu. Il garde alors 
une dimension arbitraire, son sens et ses interprétations sont ouvertes, c'est une convention. Soit une règle qui se maintient d'elle-même, parce que c'est une stratégie autorenforçante. S'il devient la définition d'un ordre social local, constitutif du fonctionnement des marchés, avec un large champ d'application, il est une institution (Bessy, Favereau, 2003). Mais il est toujours partie prenante de la régulation d'ensemble de l'économie et des divisions sociales. Les changements de schémas, leur évolution, au sein d'une gamme qui n'est pas infinie, sont toujours possibles. La crise du Covid-19 nous incite à y réfléchir.

\section{BIBLIOGRAPHIE}

Alexandre H., Buisson-Stephan H., 2014, « L'impact de la crise de 2008 sur le rationnement du crédit des PME françaises », Revue internationale des PME, 27 (2), pp.95-113.

Asensio A., Charles S, Lang D. et Le Heron E ., 2011, « Les développements récents de la macroéconomie post-keynésienne », Revue de la régulation, 10, 2e semestre .

Bartolomei A., de Oliveira M., Eloire F., Lemercier C. et Sougy N., 2017, « L'encastrement des relations entre marchands en France, 1750-1850: Une révolution dans le monde du commerce? », Annales. Histoire, Sciences Sociales, 72e année,(2), pp 425-460.

Bernardot M., 2012, Captures, Bellecombe en Bauge, Editions du Croquant, coll. Kriticos.

Bessy C., Favereau O., 2003, «Institutions et économie des conventions », Cahiers d'économie Politique / Papers in Political Economy, vol. 44, no. 1.

Bouvier J., 1960, Le Krach de l'Union générale (1878-1885), Paris, Presses universitaires de France.

Chauvin S., 2010, Les agences de la précarité. Journaliers à Chicago Paris, Le Seuil.

Cron F.,1989, Mémoires de François Cron. Déporté de la Commune Paris, Mercure de France.

Daru B., 1873, Enquête sur les actes du gouvernement de la défense nationale séance du 13 novembre 1872. Rapport de Monsieur le comte Daru, Paris, Assemblée Nationale .

De Goede M., 2005, Virtue, Fortune and Faith: A Genealogy of Finance, Minneapolis, University of Minnesota Press.

Decosse F., 2018, «De la dette au paternalisme : le bridage de la mobilité des saisonniers agricoles migrants "OFII"", in El Miri M., Mercier D., Peraldi M. (dir.), Frontières en travail : migrations, travail et fabrique des frontières dans le monde, Paris, Karthala, Terrains du siècle.

Ellman M., 2000, « The 1947 Soviet Famine and the Entitlement Approach to Famines », Cambridge Journal of Economics, 24(5), 603-630.

Etudes et Conjoncture., 1948, «La technique du rationnement et l'évolution économique », Economie mondiale, 3è année, nº-8, p. 163-193.

Fourel C., 2019, « Sortie du capitalisme, revenu d'existence et monnaies alternatives dans la pensée de Gorz », Variations [En ligne], 22. 
Garcia M-F., 1986, « La construction sociale d'un marché parfait. Le marché au cadran de Fontaines-en-Sologne ", Actes de la recherche en sciences sociales, Vol. 65, La construction sociale de l'économie, p. 2-13.

Jorion P., 2010, Le prix, Bellecombe en Bauge, Éditions du Croquant, coll. « Dynamiques socioéconomiques ", 2010.

Kaufmann L ., Quéré L., (2001), « Comment analyser les collectifs et les institutions?

Ethnométhodologie et holisme anthropologique », in de Fornel M. (dir.), L'ethnométhodologie. Une sociologie radicale, La Découverte, p. 361-390.

Lallement M., 2009, Le travail de l'utopie. Godin et le familistère de Guise, Paris, Les Belles Lettres. Laurentjoye T., Malherbe L., 2019, « Éléments institutionnalistes pour la mise en perspective historique du concept de monnaie endogène », Revue de la régulation, 26|2nd semestre / Autumn

Le Marchand A., 2018, «Économie de bazar et économie morale: crédit et caravanes ». Études Tsiganes, 64(2), p. 22-39.

Lerquet I., 2014, « Les jetons-monnaies des lieux de plaisir », Numibec, Vol n².

Lissagaray P-O.,1970, [1876], L'histoire de la commune de 1871, Paris, Petite collection Maspéro.

Malinvaud E,.1980, Réexamen de la théorie du chômage, Paris, Calmann-Lévy .

Malon C ., 2017, « Le négoce du Havre » in E. Saunier, J. Barzman (dir.), Histoire du Havre, Toulouse, Privat, p. 249-272.

Mesini B., Laurent C., 2015, « Concurrence des marchés de main-d'oeuvre et dumping social dans l'agriculture », Économie rurale, 349-350, p. 171-176.

Michel A.(2012), Le siège de Paris, journal d'un parisien 1870-1871, Paris, Arléa .

Muller S. (2008). À l'abattoir : travail et relations professionnelles face au risque sanitaire, Paris, Maison des sciences de l'homme, coll. « Natures sociales».

Noiret C.(1876), Projet de syndicat et de caisse d'échange (pour les ouvriers du port) et d'amélioration morale dans notre population, Le Havre, Imprimerie Maudot, Godfroy et Compagnie.

Norman P. (2011), The Risk Controllers: Central Counterparty Clearing in Globalised Financial Markets, Chichester, John Wiley and Sons Ltd.

Paradis J-H.(2010) [1872], Journal du siège de Paris, Septembre 1870-janvier 1871, Paris Tallandier.

PERROT, MICHELLE.1973. LES OUVRIERS EN GRÈVE :FRANCE 1870-1891. PARIS, MOUTON.

Rème Harnay P, Guerrien B., 2009, « Quelle synthèse entre économie et sociologie ? », Cahiers internationaux de sociologie $2, \mathrm{n}^{\circ} 127$, p. 339-349.

Sen A., 1977, "Starvation and exchange entitlements: a general approach and its application to the great Bengal famine", Cambridge Journal of Economics, vol. 1, no. 1.

Senn 0.,1888, Études sur les «marchés à terme » en marchandises et leur liquidation, Paris, Librairie Guillaumin.

Szuba, M, Semal L., 2010, « Rationnement volontaire contre « abondance dévastatrice » :

l'exemple des crags », Sociologies pratiques, vol. 20, no. 1, p. 87-95.

Théret B., 2008, «Les trois états de la monnaie: Approche interdisciplinaire du fait monétaire. Revue économique », vol. 59(4), p. 813-841. 
Thompson E P., 1971, « The moral economy of the english crowd, in the eigteenth century » Past \& Present,50, p70-136.

Thuillier G., 1959, « Pour une histoire monétaire de la France au XIXe siècle : le rôle des monnaies de cuivre et de billon », Annales. Economies, sociétés, civilisations. 14? année, N. 1, p. 65-90.

Topik S., 1991, «L'État sur le marché : approche comparative du café brésilien et du hennequen mexicain. ", Annales. Economies, sociétés, civilisations, 46? année, N. 2, p. 429-457, Trad. par JeanFrançois Sené.

Tricou F., 2008, La loi de l'offre et de la demande. Une enquête sur le libéralisme économique. Lille, Septentrion.

Turmann M., 1912. «Rôle économique et social des caisses de liquidation en France», Revue économique internationale, août, p. 321-355.

Vigarié A., 2003. Épidémies et commerce maritime au Havre de 1848 à 1918. Aspects de la géographie médicale du port. In: Études Normandes, 52 e année, $n^{\circ} 2,2003$. Un tour de Normandie. pp. 45-58.

Voldman D., 2016, Locataires et propriétaires. Une histoire française, Paris, Bibliothèque historique Payot.

Walter C., 2005, « Les quatre causes de l'efficacité informationnelle des marchés »,Finance \& Bien Commun, /3 No 23, p. 107-115.

Zelizer, V., 2005 [1994]. La signification sociale de l'argent, trad. par Christian Cler. Paris, Seuil, coll. «Liber» ( ed. orig. The Social Meaning of Money, New York, Basic Books).

\section{NOTES}

1. Archives du Havre FC I2, 7,15

2. Stanislas Du Guerny « Le Marché du porc breton va tenter de se relancer », Les Echos.fr, $23 / 11 / 2015$.

3. Voir les notes de synthèse hebdomadaire du marché de Plérin sur son site https:// www.marche-porc-breton.com/wp-content/uploads/nth/nth2220.pdf

4. Coronavirus outbreak exposing vulnerabilities in the EU meat sector Natasha Foote Euractiv.com 19 mai 2020

5. Ces deux articles sont de Julia Pascual.

\section{RÉSUMÉS}

La crise du covid a ramené en pleine lumière le rationnement. Il est classiquement défini comme la conséquence de rigidités des prix, mais il peut être, à l'inverse, la cause de ses rigidités. La pluralité monétaire est un moyen de rationner et discriminer certains acteurs. Le terme fut introduit lors du siège de Paris en 1870, revenir sur ces circonstances, permet de comprendre ses 
liens avec la construction sociale des marchés. Par la suite, à l'occasion d'un crédit crunch en 1882, c'est un rationnement, via une caisse de liquidation, qui permit de légitimer les marchés à terme. Des jetons sont utilisés pour payer des dockers, après des épisodes de choléra. Au $\mathrm{Xx}^{\mathrm{e}}$ siècle, les marchés physiques sont des observatoires commodes de ces règles, dont les conséquences sont très sensibles sur les travailleurs saisonniers et sur les plus vulnérables. Lors de l'actuelle pandémie, les règles de rationnement se dévoilent sur le marché du travail des saisonniers et sur les marchés.

INDEX

Mots-clés : Rationnement, Monnaie endogène, Monnaie locale, Marchés, Travail atypique

\section{AUTEUR}

\section{ARNAUD LE MARCHAND}

Maître de conférences en économie à l'Université du Havre-Normandie, Habilité à diriger des recherches en sociologie. Membre de l'UMR-CNRS-IDEES Le Havre. 